\title{
ON THE LACK OF INVERSES TO $C^{*}$-EXTENSIONS RELATED TO PROPERTY T GROUPS
}

\author{
V. MANUILOV AND K. THOMSEN
}

\begin{abstract}
Using ideas of $\mathrm{S}$. Wassermann on non-exact $C^{*}$-algebras and property $\mathrm{T}$ groups, we show that one of his examples of non-invertible $C^{*}$-extensions is not semiinvertible. To prove this, we show that a certain element vanishes in the asymptotic tensor product. We also show that a modification of the example gives a $C^{*}$-extension which is not even invertible up to homotopy.
\end{abstract}

\section{INTRODUCTION}

The Brown-Douglas-Fillmore theory of $C^{*}$-extensions, 2], works nicely for nuclear $C^{*}$ algebras because an extension of a nuclear $C^{*}$-algebra is always invertible in the extension semi-group. As a steadily growing number of examples show, this is not the case for general extensions, cf. [1],[9, [18, [17, [19], [6], [13], etc. In contrast, besides all its other merits, the $E$-theory of Connes and Higson, [3], provides a framework which incorporates arbitrary extensions of $C^{*}$-algebras, and in previous work we have clarified in which way this happens, cf. [10, 11]. Specifically, in the $E$-theory setting the notion of triviality of extensions must we weakened, at least so far as to consider an extension of $C^{*}$-algebras

$$
0 \longrightarrow B \longrightarrow E \stackrel{q}{\longrightarrow} A \longrightarrow 0
$$

to be trivial when it is asymptotically split, by which we mean that there is an asymptotic homomorphism, [3], $\varphi=\left(\varphi_{t}\right)_{t \in[0, \infty)}: A \rightarrow E$ such that $q \circ \varphi_{t}=\operatorname{id}_{A}$ for each $t \in[0, \infty)$. When the quotient $C^{*}$-algebra $A$ is a suspension, i.e. is of the form $C_{0}(\mathbb{R}) \otimes D$, this is the only change which is needed to ensure that $E$-theory becomes a complete analogue of the BDF theory for nuclear $C^{*}$-algebras. Specifically, when the quotient $C^{*}$-algebra is a suspension and the ideal is stable, every extension is semi-invertible, by which we mean that it is invertible in the sense corresponding to the weakened notion of triviality, i.e. one can add an extension to it so that the result is asymptotically split. Furthermore a given extension will represent 0 in $E$-theory if and only if it can be made asymptotically split by adding an asymptotically split extension to it. One purpose of the present paper is to show by example that this nice situation does not persist when the quotient $C^{*}$-algebra is not a suspension. We will show that an extension considered by S. Wassermann in [19], and shown by him to be non-invertible, is not semi-invertible either. By slightly modifying Wassermann's example, we obtain also an extension which is not even invertible up to homotopy, giving us the first example of a $C^{*}$-algebra for which the semi-group of homotopy classes of extensions by a stable $C^{*}$-algebra, in casu the algebra of compact operators, is not a group. The conclusion is that the $E$-theory approach to $C^{*}$-extensions does not completely save us from the unpleasantness of extensions without inverses. But unlike the BDF theory, as shown in [11, in E-theory they can be eliminated at the cost of a single suspension.

2000 Mathematics Subject Classification. Primary 19K33; Secondary 46L06, 46L80, 20 F99.

Key words and phrases. $C^{*}$-algebra extension, property T group, asymptotic tensor $C^{*}$-norm.

The first named author acknowledges partial support from RFFI, grant No. 02-01-00574, and НШ-619.2003.01. 
The method we adopt in order to show that Wassermann's example from [19] is not semi-invertible is basically the same as his, although the verification is somewhat more complicated since it uses the asymptotic tensor norm, which was introduced in [12, in place of the minimal tensor norm. To show that a suitably modified version of the extension is not even invertible up to homotopy we proceed quite differently in order to bring a $K$-theoretical obstruction to bear.

\section{WASSERMANN'S EXTENSION IS NOT SEMI-INVERTIBLE}

1.1. Wassermann's example. Let $G$ be an infinite countable discrete group with the property $\mathrm{T}$ of Kazhdan, [14. It is a result of Wang, [16], cf. [19], that there is at most a countable number of unitary equivalence classes of finite-dimensional unitary representations of $G$. As in [19], we assume that there actually are infinitely many equivalence classes of such representations. This is the case, for example, when $G=S L_{3}(\mathbb{Z})$. We fix then a sequence $\pi_{k}, k=1,2,3, \ldots$, of inequivalent finite-dimensional irreducible unitary representations of $G$ which contains a representative for each equivalence class of such representations. Consider the direct sum $\pi=\oplus_{k=1}^{\infty} \pi_{k}$ of these representations, acting on the Hilbert space $H$, and let $B$ be the $C^{*}$-subalgebra of $\mathbb{L}(H)$ generated by $\{\pi(g): g \in G\}$. The $C^{*}$-subalgebra of $\mathbb{L}(H)$ generated by $B$ and the ideal $\mathbb{K}=\mathbb{K}(H)$ of compact operators on $H$ will be denoted by $E$. Then $\mathbb{K}$ is an ideal in $E$ and we denote the quotient $E / \mathbb{K}$ by $A$. It was shown in [19] that the extension

$$
0 \longrightarrow \mathbb{K} \longrightarrow E \stackrel{q}{\longrightarrow} A \longrightarrow 0
$$

is not invertible, or not semi-split. We are going to prove that it is also not semi-invertible.

Theorem 1.1. The extension (1.1) is not semi-invertible.

We shall elaborate a little on Wassermann's argument, so let us therefore first outline it. He shows that (1.1) does not admit a completely positive section for the quotient map (i.e. is not invertible) because the sequence

$$
\mathbb{K} \otimes B \longrightarrow E \otimes_{\min } B \longrightarrow A \otimes_{\min } B
$$

is not exact. That (1.2) is not exact he deduces as follows: The representation $G \ni$ $g \mapsto \pi(g) \otimes \pi(g)$ of $G$ in $E \otimes_{\min } B$ extends by the universal property of $C^{*}(G)$ to a *-homomorphism

$$
\Delta: C^{*}(G) \rightarrow E \otimes_{\min } B .
$$

By Theorem 1.10 of [8], $G$ is finitely generated. Let $g_{1}, g_{2}, \ldots, g_{n}$ be a set of generators of $G$. We assume that this set contains the neutral element and is symmetric, i.e. contains $g_{i}^{-1}$ for all $i=1, \ldots, n$. Wassermann shows in the proof of Theorem 6 of [19] that there is $\delta>0$ such that the spectrum of the image of the element

$$
\Delta\left(\frac{1}{n} \sum_{i=1}^{n} g_{i}\right) \in E \otimes_{\min } B
$$

in the quotient $E \otimes_{\min } B / \mathbb{K} \otimes B$ lies in $[-1,1-\delta] \cup\{1\}$ and contains 1, while the spectrum of its image in $A \otimes_{\min } B$, under the quotient map of (1.2), lies in $[-1,1-\delta]$. Thus (1.2) is clearly not exact, and it follows that (1.1) is not invertible.

In order to adopt this approach in the asymptotic setting it is crucial that Wassermann's proof of Theorem 6 in [19] gives a tiny bit of additional information. Recall, [14, [7, that 1 is an isolated point in the spectrum of $\frac{1}{n} \sum_{i=1}^{n} g_{i}$ in $C^{*}(G)$, and that the corresponding spectral projection $p$ is the support projection of the trivial representation. In particular, $p=h\left(\frac{1}{n} \sum_{i=1}^{n} g_{i}\right)$ for an appropriately chosen continuous function $h$ on $[-1,1]$, and then 
it is clear that Wassermann's argument for Theorem 6 in 19] proves that the image of $p$ is non-zero in $E \otimes_{\min } B / \mathbb{K} \otimes B$, but zero in $A \otimes_{\min } B$. It is this fact, that the noninvertibility of (1.1) can be detected by the non-vanishing of a projection in a certain $C^{*}$-algebra, which makes it possible to adopt it to the asymptotic case.

1.2. Left asymptotic tensor $C^{*}$-norm. Let us review the construction of the left asymptotic tensor norm, cf. [12]. Let $\varphi=\left(\varphi_{t}\right)_{t \in[1, \infty)}: A \rightarrow \mathbb{L}\left(H_{1}\right)$ be an asymptotic homomorphism from $A$ to the $C^{*}$-algebra of bounded operators $\mathbb{L}\left(H_{1}\right)$ of some Hilbert space $H_{1}$, in the following referred to as an asymptotic representation of $A$, and let $\pi: B \rightarrow \mathbb{L}\left(H_{2}\right)$ be a (genuine) representation of $B . \varphi$ and $\pi$ define in the natural way two commuting *-homomorphisms,

$$
A \rightarrow C_{b}\left([1, \infty), \mathbb{L}\left(H_{1} \otimes H_{2}\right)\right) / C_{0}\left([1, \infty), \mathbb{L}\left(H_{1} \otimes H_{2}\right)\right)
$$

and

$$
B \rightarrow C_{b}\left([1, \infty), \mathbb{L}\left(H_{1} \otimes H_{2}\right)\right) / C_{0}\left([1, \infty), \mathbb{L}\left(H_{1} \otimes H_{2}\right)\right),
$$

giving rise to a $*$-homomorphism

$$
\varphi \odot \pi: A \odot B \rightarrow C_{b}\left([1, \infty), \mathbb{L}\left(H_{1} \otimes H_{2}\right)\right) / C_{0}\left([1, \infty), \mathbb{L}\left(H_{1} \otimes H_{2}\right)\right) .
$$

The left asymptotic tensor norm $\|\cdot\|_{\lambda}$, defined on $A \odot B$, is

$$
\|c\|_{\lambda}=\sup _{\varphi, \pi}\|\varphi \odot \pi(c)\|
$$

where the supremum is taken over all asymptotic representations of $A$ and all representations of $B$. On a linear combination, $c=\sum_{i=1}^{m} a_{i} \otimes b_{i}$, of simple tensors,

$$
\|c\|_{\lambda}=\sup _{\varphi, \pi}\left(\limsup _{t \rightarrow \infty}\left\|\sum_{i=1}^{m} \varphi_{t}\left(a_{i}\right) \otimes \pi\left(b_{i}\right)\right\|\right) \text {. }
$$

Let $A \otimes_{\lambda} B$ be the completion of $A \odot B$ with respect to the norm $\|\cdot\|_{\lambda}$.

It is a convenient fact that the left asymptotic tensor norm can be calculated using only a single representation of $B$ :

Lemma 1.2. Let $\pi^{\prime}: B \rightarrow \mathbb{L}\left(H^{\prime}\right)$ be a faithful representation of $B$ such that $\pi^{\prime}(B) \cap$ $\mathbb{K}\left(H^{\prime}\right)=\{0\}$. Then

$$
\|c\|_{\lambda}=\sup _{\varphi}\left\|\varphi \odot \pi^{\prime}(c)\right\|
$$

for all $c \in A \odot B$.

Proof. Let $\rho: B \rightarrow \mathbb{L}\left(H_{2}\right)$ be an arbitrary representation of $B$. We claim that

$$
\|\varphi \odot \rho(c)\| \leq\left\|\varphi \odot \pi^{\prime}(c)\right\|
$$

for every asymptotic representation $\varphi$ of $A$ and every $c \in A \odot B$. To prove this, let $\varepsilon>0$ and write $c=\sum_{i=1}^{m} a_{i} \otimes b_{i}$. By Voiculescu's non-commutative Weyl-von Neumann theorem, [15, there is an isometry $V: H_{2} \rightarrow H^{\prime}$ such that $\sum_{i=1}^{m}\left\|a_{i}\right\|\left\|\rho\left(b_{i}\right)-V^{*} \pi^{\prime}\left(b_{i}\right) V\right\| \leq \varepsilon$. Since $\lim \sup _{t \rightarrow \infty}\left\|\varphi_{t}\left(a_{i}\right)\right\| \leq\left\|a_{i}\right\|$, it follows that

$$
\begin{aligned}
\|\varphi \odot \rho(c)\| & \leq \limsup _{t \rightarrow \infty}\left\|\sum_{i=1}^{m} \varphi_{t}\left(a_{i}\right) \otimes V^{*} \pi^{\prime}\left(b_{i}\right) V\right\|+\varepsilon \\
& \leq \limsup _{t \rightarrow \infty}\left\|\left(1 \otimes V^{*}\right)\left(\sum_{i=1}^{m} \varphi_{t}\left(a_{i}\right) \otimes \pi^{\prime}\left(b_{i}\right)\right)\left(1 \otimes V^{*}\right)\right\|+\varepsilon \\
& \leq \limsup _{t \rightarrow \infty}\left\|\sum_{i=1}^{m} \varphi_{t}\left(a_{i}\right) \otimes \pi^{\prime}\left(b_{i}\right)\right\|+\varepsilon
\end{aligned}
$$


proving (1.3) and hence the lemma.

We show now how the asymptotic tensor norm can be used in proving non-semiinvertibility of an extension.

Note that, thanks to the exactness of the maximal tensor product, $E \otimes_{\min } B / \mathbb{K} \otimes B$ is a quotient of $A \otimes_{\max } B$. On the other hand, $A \otimes_{\min } B$ is the quotient of $E \otimes_{\min } B / \mathbb{K} \otimes B$. Therefore $A \odot B$ is a dense subspace in $E \otimes_{\min } B / \mathbb{K} \otimes B$. We denote the norm on $A \odot B$ inherited from $E \otimes_{\min } B / \mathbb{K} \otimes B$ by $\|\cdot\|_{E}$. Since this norm is a cross-norm, we may write view $E \otimes_{\min } B / \mathbb{K} \otimes B$ as a tensor product of $A$ and $B$ and write $E \otimes_{\min } B / \mathbb{K} \otimes B=A \otimes_{E} B$. Recall that $A \odot B$ is dense in $A \otimes_{\lambda} B$ as well.

Lemma 1.3. Suppose that there exists $c \in A \odot B$ such that $\|c\|_{E}>\|c\|_{\lambda}$. Then the extension (1.1) is not semi-invertible.

Proof. The idea of the proof is borrowed from [17. Suppose the contrary, i.e. that (1.1) is semi-invertible. Then there exists an extension $0 \longrightarrow \mathbb{K} \longrightarrow E^{\prime} \stackrel{q^{\prime}}{\longrightarrow} A \longrightarrow 0$ and an asymptotic splitting $s=\left(s_{t}\right)_{t \in[0, \infty)}: A \rightarrow C$, where $D \subset M_{2}(\mathbb{L}(H))$ is the $C^{*}$-subalgebra

$$
D=\left\{\left(\begin{array}{cc}
e & b_{1} \\
b_{2} & e^{\prime}
\end{array}\right): b_{1}, b_{2} \in \mathbb{K}, e \in E, e^{\prime} \in E^{\prime}, q(e)=q^{\prime}\left(e^{\prime}\right)\right\} .
$$

By definitition of the left asymptotic tensor norm there is an asymptotic homomorphism $s_{t} \otimes_{\lambda} \operatorname{id}_{B}: A \otimes_{\lambda} B \rightarrow D \otimes_{\min } B$ with the property that

$$
\lim _{t \rightarrow \infty} s_{t} \otimes_{\lambda} \operatorname{id}_{B}(a \odot b)-s_{t}(a) \odot b=0
$$

on simple tensors. Let $d: D \rightarrow E$ be the completely positive contraction given by $d\left(\begin{array}{cc}e & b_{1} \\ b_{2} & e^{\prime}\end{array}\right)=e$. Then the map $d \otimes \operatorname{id}_{B}: D \otimes_{\min } B \rightarrow E \otimes_{\min } B$ is a well-defined contraction. Let $q_{B}: E \otimes_{\min } B \rightarrow E \otimes_{\min } B / \mathbb{K} \otimes B=A \otimes_{E} B$ be the quotient map. Consider the composition

$$
r_{t}=q_{B} \circ\left(d \otimes \operatorname{id}_{B}\right) \circ\left(s_{t} \otimes_{\lambda} \operatorname{id}_{B}\right): A \otimes_{\lambda} B \rightarrow A \otimes_{E} B .
$$

The maps $q_{B}$ and $d \otimes \mathrm{id}_{B}$ are contractions and the family $\left(s_{t} \otimes_{\lambda} \mathrm{id}_{B}\right)_{t \in[1, \infty)}$ is asymptotically contractive, so the family $\left(r_{t}\right)_{t \in[1, \infty)}$ is asymptotically contractive. Since $\lim _{t \rightarrow \infty} r_{t}(c)-c=$ 0 , it follows that $\|c\|_{E}=\lim \sup _{t \rightarrow \infty}\left\|r_{t}(c)\right\|_{E} \leq\|c\|_{\lambda}$. The contradiction to $\|c\|_{E}>\|c\|_{\lambda}$ completes the proof.

Let $f(t)$ be a polynomial $f(t)=\frac{1}{4} t^{2}+\frac{1}{2} t+\frac{1}{4}$, and set $x=f\left(\frac{1}{n} \sum_{i=1}^{n} g_{i}\right) \in C^{*}(G)$. Then $0 \leq x \leq 1$ and 1 is an isolated point in the spectrum of $x$. Put $\Delta(x) \in A \odot B$. As pointed out above, Wassermann has shown that the spectrum of the element $\Delta\left(\frac{1}{n} \sum_{i=1}^{n} g_{i}\right) \in A \odot B$ in the quotient $E \otimes_{\min } B / \mathbb{K} \otimes B$ contains 1 , and it follows that $\|\Delta(x)\|_{E}=1$. By Lemma 1.3. Theorem 1.1 will follow if we show that

$$
\|\Delta(x)\|_{\lambda}<1 .
$$

Let $\Delta_{\lambda}: C^{*}(G) \rightarrow A \otimes_{\lambda} B$ be the $*$-homomorphism determined by the condition that $\Delta_{\lambda}(g)=q(\pi(g)) \otimes \pi(g), g \in G$. The desired conclusion, (1.4), is then equivalent to

$$
\left\|\Delta_{\lambda}(p)\right\|_{\lambda}=0
$$

because 1 is isolated in the spectrum of $x$. 


\subsection{Calculation of $\left\|\Delta_{\lambda}(p)\right\|_{\lambda}$.}

Lemma 1.4. One has $\left\|\Delta_{\lambda}(p)\right\|_{\lambda}=0$.

Proof. Set $H^{\prime}=\oplus_{i=1}^{\infty} H$ and let $i_{\infty}: B \rightarrow \mathbb{L}\left(H^{\prime}\right)$ be the infinite sum of copies of the inclusion $B \subseteq \mathbb{L}(H)$. Then

$$
\|c\|_{\lambda}=\sup _{\varphi}\left\|\varphi \odot i_{\infty}(c)\right\|
$$

for all $c \in A \odot B$ by Lemma 1.2. Let $\varepsilon \in\left(0, \frac{1}{100}\right)$. There is then an asymptotic representation $\varphi: A \rightarrow \mathbb{L}\left(H_{1}\right)$ and an equi-continuous asymptotic representation $\Phi: A \otimes_{\lambda} B \rightarrow$ $\mathbb{L}\left(H_{1} \otimes H^{\prime}\right)$ such that

$$
\limsup _{t \rightarrow \infty}\left\|\Phi_{t}\left(\Delta_{\lambda}(p)\right)\right\| \geq\left\|\Delta_{\lambda}(p)\right\|_{\lambda}-\varepsilon
$$

and

$$
\lim _{t \rightarrow \infty}\left\|\Phi_{t}(c)-\sum_{i=1}^{m} \varphi_{t}\left(a_{i}\right) \otimes i_{\infty}\left(b_{i}\right)\right\|=0
$$

for all $c=\sum_{i=1}^{m} a_{i} \otimes b_{i} \in A \odot B$. For each $k$, let $q_{k}^{\prime}$ be the orthogonal projection onto the support in $H$ of the representation $\pi_{k}$, and let $q_{k}=\prod_{i=1}^{\infty} q_{k}^{\prime} \in \mathbb{L}\left(H^{\prime}\right)$ be the infinite repeat of $q_{k}^{\prime}$. Note that each $1_{H_{1}} \otimes q_{k}$ commutes with $\sum_{i=1}^{m} \varphi_{t}\left(a_{i}\right) \otimes i_{\infty}\left(b_{i}\right)$ for all $t$ and all $c=\sum_{i=1}^{m} a_{i} \otimes b_{i} \in A \odot B$. By approximating $\Delta_{\lambda}(p)$ with elements from $A \odot B$ we can find an element $z=\sum_{i=1}^{m} a_{i} \otimes b_{i} \in A \odot B$ such that

$$
\limsup _{t \rightarrow \infty}\left\|\Phi_{t}\left(\Delta_{\lambda}(p)\right)-\sum_{i=1}^{m} \varphi_{t}\left(a_{i}\right) \otimes i_{\infty}\left(b_{i}\right)\right\|<\varepsilon .
$$

To simplify notation, set $z_{t}=\sum_{i=1}^{m} \varphi_{t}\left(a_{i}\right) \otimes i_{\infty}\left(b_{i}\right)$, and $y_{t}=\frac{1}{2}\left(z_{t}+z_{t}^{*}\right)$. Since $\Phi$ is an asymptotic homomorphism and $\Delta_{\lambda}(p)$ a projection it follows from (1.7) that for some $T>0$

when $t \geq T$. It follows that

$$
\left\|y_{t}^{2}-y_{t}\right\| \leq 5 \varepsilon
$$

$$
\left\|\left(\left(1_{H_{1}} \otimes q_{k}\right) y_{t}\right)^{2}-\left(1_{H_{1}} \otimes q_{k}\right) y_{t}\right\| \leq 5 \varepsilon
$$

for all $t>T$. Since $5 \varepsilon<1 / 4$, we find that the characteristic function $h=1_{[1 / 2, \infty)}$ is continuous on the spectrum of $y_{t}$ and on the spectrum of each $\left(1_{H_{1}} \otimes q_{k}\right) y_{t}$ when $t>T$. It follows that $h\left(y_{t}\right)$ and $h\left(\left(1_{H_{1}} \otimes q_{k}\right) y_{t}\right)$ are projections for all $k$ and all $t>T$. We claim that

$$
h\left(y_{t}\right)=0
$$

for all $t>T$. If not there is some $t_{0}>T$ such that $h\left(y_{t_{0}}\right) \neq 0$. There must then be a $k$, which we now fix, such that $h\left(\left(1_{H_{1}} \otimes q_{k}\right) y_{t_{0}}\right) \neq 0$ since $\sum_{i} 1_{H_{1}} \otimes q_{i}=1$. But then

$$
\left\|h\left(\left(1_{H_{1}} \otimes q_{k}\right) y_{t}\right)\right\|=1
$$

for all $t>T$ since $h\left(\left(1_{H_{1}} \otimes q_{k}\right) y_{t}\right)$ varies norm-continuously with $t$ and is a projection for all $t>T$. Let $\rho_{k}: B \rightarrow C^{*}\left(\pi_{n}(G)\right)$ be the finite-dimensional representation of $B$ obtained by restricting the elements of $B$ to the subspace of $H$ supporting the representation $\pi_{k}$ of $G$. There is then a representation $\mu: C^{*}\left(\pi_{n}(G)\right) \rightarrow \mathbb{L}\left(H^{\prime}\right)$ such that

$$
\mu \circ \rho_{k}(b)=q_{k} i_{\infty}(b)
$$

for all $b \in B$. Furthermore, there is an equi-continuous asymptotic homomorphism $\psi$ : $A \otimes C^{*}\left(\pi_{n}(G)\right) \rightarrow \mathbb{L}\left(H_{1} \otimes H^{\prime}\right)$ such that

$$
\lim _{t \rightarrow \infty}\left\|\psi_{t}(c)-\sum_{i=1}^{m} \varphi_{t}\left(a_{i}\right) \otimes \mu\left(x_{i}\right)\right\|=0
$$


for all $c=\sum_{i=1}^{m} a_{i} \otimes x_{i} \in A \odot C^{*}\left(\pi_{n}(G)\right)$. Note that $\operatorname{id}_{A} \otimes \rho_{k}: A \odot B \rightarrow A \odot C^{*}\left(\pi_{n}(G)\right)$ extends to a $*$-homomorphism $\kappa: A \otimes_{\lambda} B \rightarrow A \otimes C^{*}\left(\pi_{n}(G)\right)$. It follows from (1.6), (1.11) and (1.12) that

$$
\lim _{t \rightarrow \infty}\left\|\psi_{t} \circ \kappa(d)-\left(1_{H_{1}} \otimes q_{k}\right) \Phi_{t}(d)\right\|=0
$$

for all $d \in A \otimes_{\lambda} B$. Since $\kappa$ factors through $A \otimes_{\min } B$, we know from [19] that $\left\|\kappa\left(\left(\Delta_{\lambda}(p)\right)\right)\right\|=$ 0 . It follows therefore from (1.13) that

$$
\limsup _{t \rightarrow \infty}\left\|\left(1_{H_{1}} \otimes q_{k}\right) \Phi_{t}\left(\left(\Delta_{\lambda}(p)\right)\right)\right\|=0,
$$

and then by use of (1.7) that

$$
\limsup _{t \rightarrow \infty}\left\|\left(1_{H_{1}} \otimes q_{k}\right) y_{t}\right\| \leq \varepsilon
$$

Since $\varepsilon<1 / 2$, this contradicts (1.10), and we conclude that (1.9) must hold. Combined with (1.8) we find that the spectrum of $y_{t}$ is contained in $[-1 / 2,1 / 2]$, and hence that $\left\|y_{t}\right\| \leq 1 / 2$. It follows then from (1.7) that

$$
\limsup _{t \rightarrow \infty}\left\|\Phi_{t}\left(\Delta_{\lambda}(p)\right)\right\| \leq 1 / 2+\varepsilon<1 .
$$

Since $\Delta_{\lambda}(p)$ is a projection, we deduce first that $\lim _{\sup _{t \rightarrow \infty}}\left\|\Phi_{t}\left(\Delta_{\lambda}(p)\right)\right\|=0$, and then from (1.5) that $\left\|\Delta_{\lambda}(p)\right\|_{\lambda}=0$.

1.4. Some remarks. Theorem 1.1 means that it is not possible to add an extension of $A$ by $\mathbb{K}$ to (1.1) such that the resulting extension admits an asymptotic homomorphism consisting of sections for the quotient map. In particular, the extension (1.1) itself does not admit such a family of sections; a fact, which may seem slightly surprising because the extension is clearly quasi-diagonal and there is an obvious sequence $s_{n}: A \rightarrow E$, $n=1,2, \ldots$, of maps, each of which is a section for the quotient map such that they form a discrete asymptotic homomorphism. It was therefore no coincidence that the connectedness of the parameter space, $[0, \infty)$, was used at a crucial point in the proof above.

In 12 we raised the question, if the left asymptotic tensor product is associative. It follows from Lemma 1.4 that the answer is negative.

We have looked through all known (to us) examples of non-invertible extensions to check if they are semi-invertible or not. The examples of Kirchberg, 9], are semi-invertible by results of [11. Another example of Wassermann, [18, can be shown to not to be semi-invertible by the same method as here. Unfortunately, we know nothing about semiinvertibility of other examples.

\section{Homotopy NON-INVERTIBILITY}

2.1. A modification of the Wassermann's extension. To give an example of an extension which is not only not semi-invertible, but also not even homotopy invertible, we modify the extension (1.1) as follows. Let $d_{i}$ be the dimension of the Hilbert space $H_{i}$ on which the representation $\pi_{i}$ acts. Let $n_{i}$ be a sequence of integers such that $\lim _{i \rightarrow \infty} \frac{n_{i}}{d_{i}}=\infty$. For each $i$ we let $n_{i} \cdot \pi_{i}$ be the direct sum of $n_{i}$ copies of the representation $\pi_{i}$, and let

$$
\pi^{\prime}=\bigoplus_{i=1}^{\infty} n_{i} \cdot \pi_{i}
$$

be the direct sum of the resulting sequence of representations, acting on the Hilbert space $H$. Let $E^{\prime}$ be the $C^{*}$-subalgebra of $\mathbb{L}(H)$ generated by $\left\{\pi^{\prime}(g): g \in G\right\}$ and by $\mathbb{K}$, the compact operators on $H$. Set $A^{\prime}=E^{\prime} / \mathbb{K}$. 
Theorem 2.1. The extension

$$
0 \longrightarrow \mathbb{K} \longrightarrow E^{\prime} \longrightarrow A^{\prime} \longrightarrow 0
$$

is not invertible in $\operatorname{Ext}_{h}\left(A^{\prime}, \mathbb{K}\right)$.

Proof. To show that (2.1) is not invertible up to homotopy, let $\varphi: A^{\prime} \rightarrow Q(\mathbb{K})$ be the Busby invariant of (2.1), and assume to reach a contradiction that $\psi: A^{\prime} \rightarrow Q(\mathbb{K})$ is an extension such that $\varphi \oplus \psi$ is homotopic to 0 . Let $V_{1}, V_{2}$ be isometries in $\mathbb{L}(H)$ such that $V_{1} V_{1}^{*}+V_{2} V_{2}^{*}=1$, and set $\lambda(a)=\operatorname{Ad} q\left(V_{1}\right) \circ \varphi(a)+\operatorname{Ad} q\left(V_{2}\right) \circ \psi(a), a \in A^{\prime}$, where $q: \mathbb{L}(H) \rightarrow Q(\mathbb{K})$ is the quotient map. There is then a commuting diagram

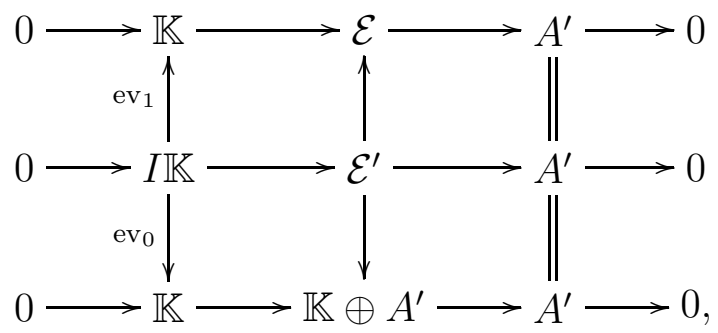

where $\lambda$ is a the Busby invariant of the upper extension, $I \mathbb{K}=C[0,1] \otimes \mathbb{K}$ and ev $_{s}: I \mathbb{K} \rightarrow$ $\mathbb{K}$ is evaluation at $s \in[0,1]$. Set $D=\prod_{k=1}^{\infty} \mathbb{L}\left(H_{k}\right)$. By tensoring with $D$ we obtain from (2.2) the commuting diagram

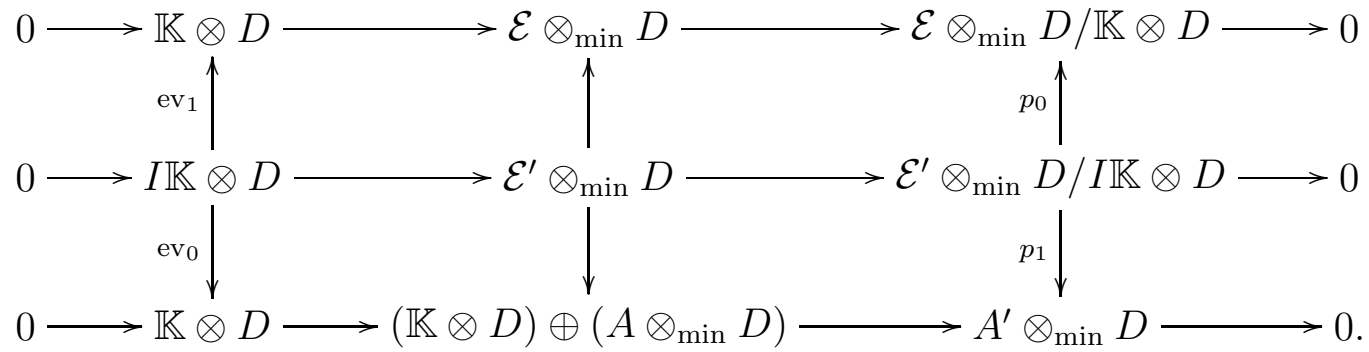

Let $\overline{\mathrm{ev}}_{s}: M(I \mathbb{K}) \rightarrow M(\mathbb{K})$ and $\widehat{\mathrm{ev}}_{s}: Q(I \mathbb{K}) \rightarrow Q(\mathbb{K})$ be the $*$-homomorphisms induced by $\mathrm{ev}_{s}$. Denote $\mathcal{E} \otimes_{\min } D / \mathbb{K} \otimes D$ and $\mathcal{E}^{\prime} \otimes_{\min } D / I \mathbb{K} \otimes D$ by $A^{\prime} \otimes_{\mathcal{E}} D$ and $A^{\prime} \otimes_{\mathcal{E}^{\prime}} D$, respectively. The Busby invariant of the middle extension of (2.3) is a $*$-homomorphism $\varphi^{\prime}: A^{\prime} \otimes_{\mathcal{E}^{\prime}} D \rightarrow Q(I \mathbb{K} \otimes D)$ such that $\widehat{\mathrm{ev}}_{1} \circ \varphi^{\prime}=\mu \circ p_{0}$, where $\mu: A^{\prime} \otimes_{\mathcal{E}} D \rightarrow Q(I \mathbb{K} \otimes D)$ is the Busby invariant of the upper extension in (2.3), while $\widehat{\mathrm{ev}}_{0} \circ \varphi^{\prime}=0$.

By the Bartle-Graves selection theorem there are continuous sections $\chi: Q(I \mathbb{K} \otimes D) \rightarrow$ $M(I \mathbb{K} \otimes D)$ and $\chi_{k}: Q\left(I \mathbb{K} \otimes D_{k}\right) \rightarrow M\left(I \mathbb{K} \otimes D_{k}\right)$ for the quotient maps $M(I \mathbb{K} \otimes D) \rightarrow$ $Q(I \mathbb{K} \otimes D)$ and $M\left(I \mathbb{K} \otimes D_{k}\right) \rightarrow Q\left(I \mathbb{K} \otimes D_{k}\right)$, respectively, for all $k=1,2,3, \ldots$ We can choose these maps to be self-adjoint and such that $\|\chi(x)\| \leq 2\|x\|, x \in Q(I \mathbb{K} \otimes D)$, and $\left\|\chi_{k}(y)\right\| \leq 2\|y\|, y \in Q\left(I \mathbb{K} \otimes D_{k}\right)$, for all $k$. Set $D_{k}=\mathbb{L}\left(H_{k}\right)$, and let $p_{k}: D=\prod_{k=1}^{\infty} D_{k} \rightarrow$ $D_{k}$ be the canonical projection. The map $\operatorname{id}_{A} \otimes p_{k}: A^{\prime} \odot D \rightarrow A^{\prime} \odot D_{k}$ extends to a $*-$ homomorphism id $\operatorname{s}_{A} \otimes p_{k}: A^{\prime} \otimes_{\mathcal{E}^{\prime}} D \rightarrow A^{\prime} \otimes D_{k}$. Let $\overline{\mathrm{id}_{I \mathbb{K}} \otimes p_{k}}: M(I \mathbb{K} \otimes D) \rightarrow M\left(I \mathbb{K} \otimes D_{k}\right)$ be the unique $*$-homomorphism extending $\operatorname{id}_{I \mathbb{K}} \otimes p_{k}: I \mathbb{K} \otimes D \rightarrow I \mathbb{K} \otimes D_{k}$, and $\widehat{\mathrm{id}}_{I \mathbb{K}} \otimes p_{k}$ : $Q(I \mathbb{K} \otimes D) \rightarrow Q\left(I \mathbb{K} \otimes D_{k}\right)$ the resulting $*$-homomorphism. Let $\Phi: A^{\prime} \rightarrow Q(I \mathbb{K})$ be the Busby invariant of the middle extension of (2.2).

We denote by $\Phi \hat{\otimes} \mathrm{id}_{D_{k}}$ the $*$-homomorphism $A^{\prime} \otimes D_{k} \rightarrow Q\left(I \mathbb{K} \otimes D_{k}\right)$ obtained by composing $\Phi \otimes \operatorname{id}_{D_{k}}: A^{\prime} \otimes D_{k} \rightarrow Q(I \mathbb{K}) \otimes D_{k}$ with the canonical embedding $Q(I \mathbb{K}) \otimes D_{k} \subseteq$ $Q\left(I \mathbb{K} \otimes D_{k}\right)$. By checking on simple tensors one finds that

$$
\mathrm{id}_{I \mathbb{K}} \otimes p_{k} \circ \varphi^{\prime}=\left(\Phi \hat{\otimes} \operatorname{id}_{D_{k}}\right) \circ\left(\operatorname{id}_{A^{\prime}} \otimes p_{k}\right),
$$


which implies that

$$
\left(\overline{\mathrm{id}_{I \mathbb{K}} \otimes p_{k}}\right) \circ \chi \circ \varphi^{\prime}(x)-\chi_{k} \circ\left(\Phi \hat{\otimes} \mathrm{id}_{D_{k}}\right) \circ\left(\operatorname{id}_{A^{\prime}} \otimes p_{k}\right)(x) \in I \mathbb{K} \otimes D_{k}
$$

for all $k$ and all $x \in A \otimes_{\mathcal{E}^{\prime}} D$. Let $\overline{\pi_{i}}$ be the representation of $G$ contragredient to $\pi_{i}$. The representation $g \mapsto q\left(\pi^{\prime}(g)\right) \otimes\left(\prod_{i=1}^{\infty} \bar{\pi}_{i}\right)(g)$ of $G$ into $A^{\prime} \odot D$ gives rise to a *homomorphism $\Delta^{\prime}: C^{*}(G) \rightarrow A^{\prime} \otimes_{\mathcal{E}^{\prime}} D$. Set $Q=\Delta^{\prime}(p)$, where $p$, as above, is the spectral projection of the element $\frac{1}{n} \sum_{i=1}^{n} g_{i}$ corresponding to the set $\{1\}$. Since $\pi_{i}$ is inequivalent to $\pi_{k}$ for all except finitely many $i$, it follows from [19], Lemma 1, that $\operatorname{id}_{A^{\prime}} \otimes p_{k}(Q)=0$ for all $k$. It follows therefore from (2.4) that

$$
\left(\overline{\mathrm{id}_{I \mathbb{K}} \otimes p_{k}}\right) \circ \chi \circ \varphi^{\prime}(Q) \in I \mathbb{K} \otimes D_{k}
$$

for all $k$. Let $P_{I}$ and $P$ denote the $*$-homomorphisms $P_{I}=\prod_{i=1}^{\infty} \overline{\operatorname{id}_{I \mathbb{K}} \otimes p_{k}}: M(I \mathbb{K} \otimes D) \rightarrow$ $\prod_{i=1}^{\infty} M\left(I \mathbb{K} \otimes D_{k}\right)$ and $P=\prod_{i=1}^{\infty} \overline{\mathrm{id}_{\mathbb{K}} \otimes p_{k}}: M(\mathbb{K} \otimes D) \rightarrow \prod_{i=1}^{\infty} M\left(\mathbb{K} \otimes D_{k}\right)$, respectively. Put

$$
N_{I}=P_{I}^{-1}\left(\prod_{i=1}^{\infty} I \mathbb{K} \otimes D_{k}\right) \subset M(I \mathbb{K} \otimes D)
$$

and

$$
N=P^{-1}\left(\prod_{i=1}^{\infty} \mathbb{K} \otimes D_{k}\right) \subset M(\mathbb{K} \otimes D) .
$$

It follows from (2.5) that

$$
\chi \circ \varphi^{\prime}(Q) \in N_{I} .
$$

Note that $I \mathbb{K} \otimes D$ is an ideal in $N_{I}$ and $\mathbb{K} \otimes D$ is an ideal in $N$. We denote the quotients $N_{I} / I \mathbb{K} \otimes D$ and $N / \mathbb{K} \otimes D$ by $R_{I}$ and $R$, respectively. Note that $R_{I} \subseteq Q(I \mathbb{K} \otimes D)$ and that $\varphi^{\prime}(Q) \in R_{I}$. Evaluation at $s \in[0,1]$ induces a $*$-homomorphism $E_{s}: M(I \mathbb{K} \otimes D) \rightarrow$ $M(\mathbb{K} \otimes D)$ with the property that $E_{s}\left(N_{I}\right)=N$, so we get a $*$-homomorphism $\widehat{E}_{s}: R_{I} \rightarrow$ $R$ induced by $E_{s}$ for each $s \in[0,1]$. To proceed with the proof, we need some calculations in $K$-theory.

2.2. $K$-theory calculations. Consider the extensions

$$
0 \longrightarrow I \mathbb{K} \otimes D \longrightarrow N_{I} \longrightarrow R_{I} \longrightarrow 0
$$

and

$$
0 \longrightarrow \mathbb{K} \otimes D \longrightarrow N \longrightarrow R \longrightarrow 0
$$

The map $\prod_{i=1}^{\infty} p_{i_{*}}: K_{0}(I \mathbb{K} \otimes D) \rightarrow \prod_{i=1}^{\infty} K_{0}\left(I \mathbb{K} \otimes D_{i}\right)$ is injective by Lemma 3.2 of [4] and the map $\prod_{i=1}^{\infty} p_{i_{*}}: K_{1}(I \mathbb{K} \otimes D) \rightarrow \prod_{i=1}^{\infty} K_{1}\left(I \mathbb{K} \otimes D_{i}\right)$ is injective by Lemma 3.3 of [4. In particular, $K_{1}(I \mathbb{K} \otimes D)=0$. Therefore the extension (2.6) gives us a commuting diagram

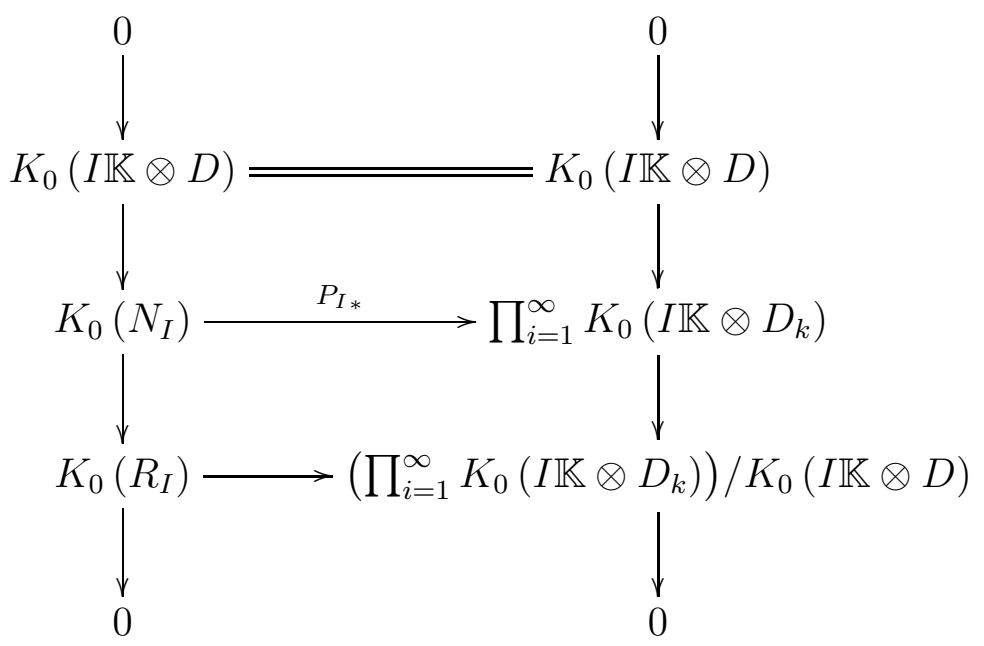


Throwing away the interval, we get also a commuting diagram

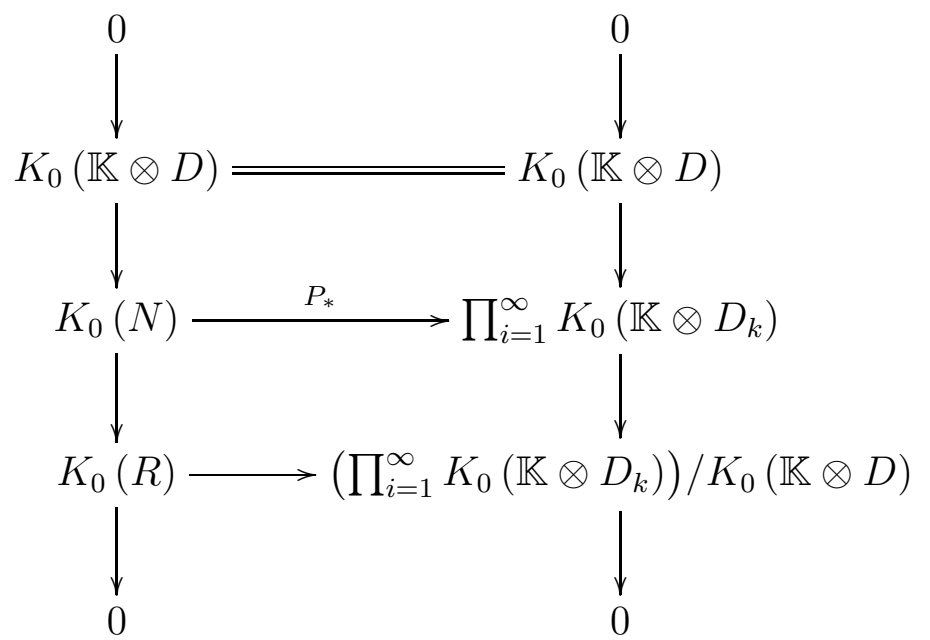

Evaluation at any $s \in[0,1]$ induces an isomorphism

$$
\left(\prod_{i=1}^{\infty} K_{0}\left(I \mathbb{K} \otimes D_{k}\right)\right) / K_{0}(I \mathbb{K} \otimes D) \rightarrow\left(\prod_{i=1}^{\infty} K_{0}\left(\mathbb{K} \otimes D_{k}\right)\right) / K_{0}(\mathbb{K} \otimes D)
$$

in the obvious way and the diagram

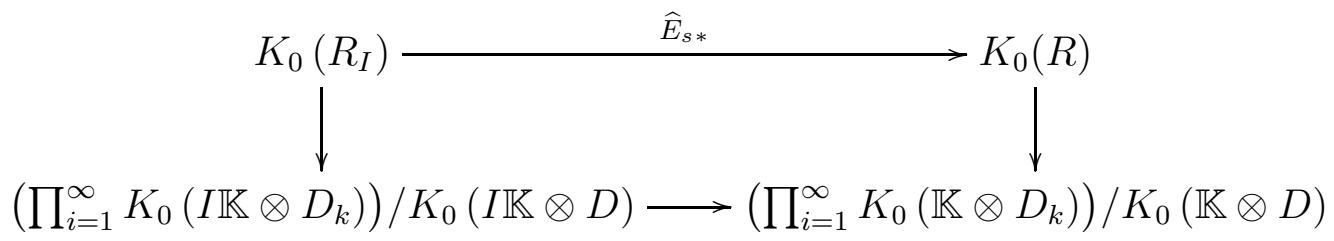

commutes for every $s \in[0,1]$. Let $x$ be the image in $\left(\prod_{i=1}^{\infty} K_{0}\left(\mathbb{K} \otimes D_{k}\right)\right) / K_{0}(\mathbb{K} \otimes D)$ of the element $\left[\varphi^{\prime}(Q)\right] \in K_{0}\left(R_{I}\right)$. Since $\widehat{\mathrm{ev}}_{0} \circ \varphi^{\prime}=0$, we conclude that $\widehat{E}_{0}\left(\varphi^{\prime}(Q)\right)=0$, which leads to the conclusion that

$$
x=0 .
$$

As we shall see, we get a different result when we consider the case $s=1$. Let $r: N \rightarrow R$ be the quotient map. Set $W_{i}=V_{i} \otimes 1_{D} \in M(\mathbb{K} \otimes D), i=1,2$. Then

$$
\widehat{E}_{1} \circ \varphi^{\prime}(Q)=r\left(W_{1} e W_{1}^{*}+W_{2} a W_{2}^{*}\right),
$$

where $e$ is the spectral projection of $\frac{1}{n} \sum_{i=1}^{n} \pi^{\prime}\left(g_{i}\right) \otimes\left(\oplus_{k=1}^{\infty} \bar{\pi}_{k}\right)\left(g_{i}\right)$ corresponding to $\{1\}$, and $a \geq 0$ is some lift in $N$ of a projection in $R \subset Q(\mathbb{K} \otimes D)$. Since $W_{i} N \subset N$ and $W_{i}^{*} N \subset N$, the $W_{i}$ 's define multipliers, first of $N$, and then of $R$. It follows therefore from (2.9) that

$$
\left[\widehat{E}_{1} \circ \varphi^{\prime}(Q)\right]=[r(e)]+[r(a)]
$$

in $K_{0}(R)$.

Consider the extension

$$
0 \longrightarrow \mathbb{K} \otimes D \longrightarrow N^{+} \stackrel{r^{+}}{\longrightarrow} R^{+} \longrightarrow 0
$$

obtained from the extension (2.7) by unitalizing. It follows from Lemma 9.6 of [5] that there are natural numbers $n, m$ such that $r(a) \oplus 1_{n} \oplus 0_{m} \in M_{1+n+m}\left(R^{+}\right)$can be lifted to a projection $f_{1} \in M_{1+n+m}\left(N^{+}\right)$. Note that the image of $f_{1}$ in $M_{1+n+m}(\mathbb{C})$ under the canonical surjection $M_{1+n+m}\left(N^{+}\right) \rightarrow M_{1+n+m}(\mathbb{C})$ is a projection of rank $n$. (We use here that the proof in [5] works equally well when the assumption that the ideal is AF is 
replaced by the weaker assumption, valid in (2.10), that the ideal has trivial $K_{1}$-group.) There is a commuting diagram

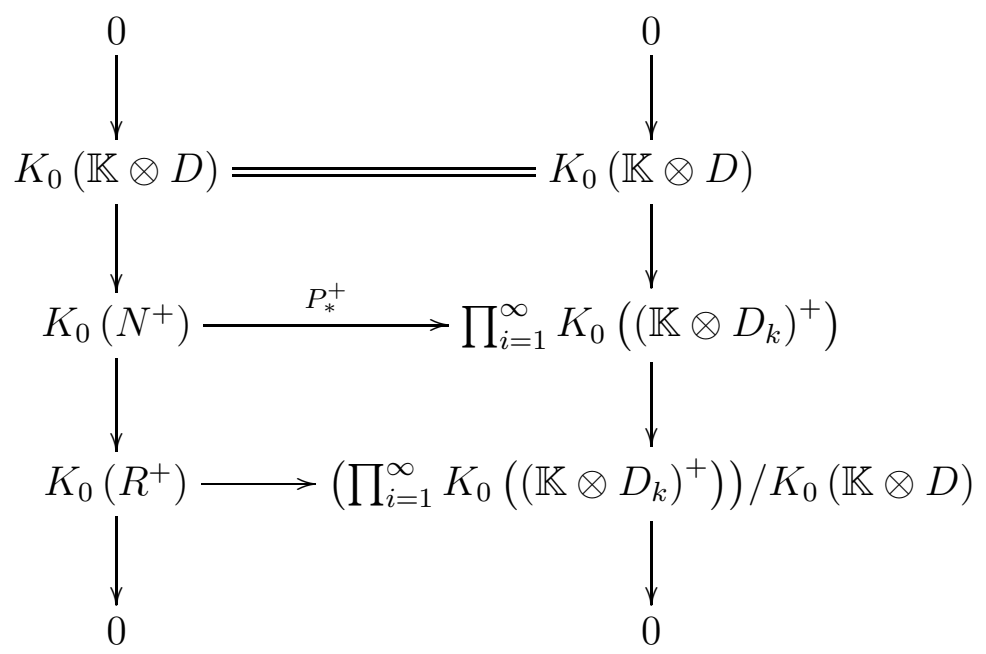

with exact columns. Thus the image of $x$ in $\left(\prod_{i=1}^{\infty} K_{0}\left(\left(\mathbb{K} \otimes D_{k}\right)^{+}\right)\right) / K_{0}(\mathbb{K} \otimes D)$ under the inclusion

$$
\left(\prod_{i=1}^{\infty} K_{0}\left(\mathbb{K} \otimes D_{k}\right)\right) / K_{0}(\mathbb{K} \otimes D) \subseteq\left(\prod_{i=1}^{\infty} K_{0}\left(\left(\mathbb{K} \otimes D_{k}\right)^{+}\right)\right) / K_{0}(\mathbb{K} \otimes D)
$$

is also the image of $P_{*}^{+}\left([e]+\left[f_{1}\right]-\left[1_{n}\right]\right) \in \prod_{i=1}^{\infty} K_{0}\left(\left(\mathbb{K} \otimes D_{k}\right)^{+}\right)$under the quotient map

$$
\prod_{i=1}^{\infty} K_{0}\left(\left(\mathbb{K} \otimes D_{k}\right)^{+}\right) \rightarrow\left(\prod_{i=1}^{\infty} K_{0}\left(\left(\mathbb{K} \otimes D_{k}\right)^{+}\right)\right) / K_{0}(\mathbb{K} \otimes D) .
$$

Write $P^{+}\left(f_{1}\right)=\left(g_{i}\right)$, where $g_{i} \in M_{1+n+m}\left(\left(\mathbb{K} \otimes D_{i}\right)^{+}\right)$for each $i$ is a projection whose image in $M_{1+n+m}(\mathbb{C})$ under the canonical surjection $M_{1+n+m}\left(\left(\mathbb{K} \otimes D_{i}\right)^{+}\right) \rightarrow M_{1+n+m}(\mathbb{C})$ is a projection of rank $n$. Since $\bigcup_{j} M_{1+n+m}\left(\left(M_{j}(\mathbb{C}) \otimes D_{i}\right)^{+}\right)$is dense in $M_{1+n+m}\left(\left(\mathbb{K} \otimes D_{i}\right)^{+}\right)$, there is a $j \in \mathbb{N}$ and a projection $f_{2}^{i} \in M_{1+n+m}\left(\left(M_{j}(\mathbb{C}) \otimes D_{i}\right)^{+}\right)$which is unitarily equivalent to $g_{i}$. Since

$$
M_{1+n+m}\left(\left(M_{j}(\mathbb{C}) \otimes D_{i}\right)^{+}\right)=M_{1+n+m}\left(M_{j}(\mathbb{C}) \otimes D_{i}\right) \oplus M_{1+n+m}(\mathbb{C}),
$$

we see that $f_{2}^{i}=f_{3}^{i}+f_{4}^{i}$, where $f_{3}^{i}$ and $f_{4}^{i}$ are orthogonal projections in $M_{1+n+m}\left(\left(\mathbb{K} \otimes D_{i}\right)^{+}\right)$, $f_{3}^{i} \in M_{1+n+m}\left(\mathbb{K} \otimes D_{i}\right)$, and $\left[f_{4}^{i}\right]=\left[1_{n}\right]$ in $K_{0}\left(\left(\mathbb{K} \otimes D_{i}\right)^{+}\right)$. It follows that

$$
P_{*}^{+}\left([e]+\left[f_{1}\right]-\left[1_{n}\right]\right)=P_{*}[e]+\left(\left[f_{3}^{i}\right]\right)_{i=1}^{\infty} \in \prod_{i=1}^{\infty} K_{0}\left(\mathbb{K} \otimes D_{k}\right) .
$$

We identify now $K_{0}\left(\mathbb{K} \otimes D_{k}\right)$ with $\mathbb{Z}$ as ordered groups, and consequently $\prod_{i=1}^{\infty} K_{0}\left(\mathbb{K} \otimes D_{k}\right)$ with $\prod_{i=1}^{\infty} \mathbb{Z}$. Then $P_{*}([e])=\left(a_{i}\right)_{i=1}^{\infty}$ and $\left(\left[f_{3}^{i}\right]\right)_{i=1}^{\infty}=\left(b_{i}\right)_{i=1}^{\infty}$, where $b_{i} \geq 0$ for all $i$, and $a_{i}$ is greater or equal to the multiplicity of the trivial representation of $G$ in $\pi^{\prime} \otimes \overline{\pi_{i}}$ which equals $n_{i}$. Since $K_{0}(\mathbb{K} \otimes D)$ is the subgroup of $\prod_{i=1}^{\infty} \mathbb{Z}$ consisting of the sequences $\left(c_{i}\right)$ in $\mathbb{Z}$ for which $\sup _{i}\left|\frac{c_{i}}{d_{i}}\right|<\infty$ by Lemma 3.2 of [4], we conclude that $x \neq 0$ because $\lim _{i \rightarrow \infty} \frac{n_{i}}{d_{i}}=\infty$. This contradicts (2.8).

The first named author is grateful to S. Wassermann for sending him a copy of his book 20]. 


\section{REFERENCES}

[1] J. Anderson, $A C^{*}$-algebra A for which $\operatorname{Ext}(A)$ is not a group, Ann. Math. 107 (1978), 455-458.

[2] L. G. Brown, R. G. Douglas, P. A. Fillmore, Extensions of $C^{*}$-algebras and K-homology, Ann. Math. 105 (1977), 265-324.

[3] A. Connes, N. Higson, Déformations, morphismes asymptotiques et K-théorie bivariante, C. R. Acad. Sci. Paris Sér. I Math. 311 (1990), 101-106.

[4] M. Dadarlat and S. Eilers, On the classification of nuclear $C^{*}$-algebras, Proc. London Math. Soc. (3) 85 (2002), 168-210.

[5] E. G. Effros, Dimensions and $C^{*}$-algebras, CBMS 46, American Mathematical Society, Providence, Rhode Island, 1981.

[6] U. Haagerup, S. Thorbjornsen, A new application of random matrices: $\operatorname{Ext}\left(C_{\mathrm{red}}^{*}\left(F_{2}\right)\right)$ is not a group, preprint, 2002, to appear in Ann. Math.

[7] P. de la Harpe, A. G. Robertson, A. Valette, On the spectrum of the sum of generators for a finitely generated group, Israel J. Math. 81 (1993), 65-96.

[8] P. de la Harpe, A. Valette, La propriété (T) de Kazhdan pour les groupes localement compacts, Astérisque, 175.

[9] E. Kirchberg, On semi-split extensions, tensor products and exactness of $C^{*}$-algebras, Invent. Math. 112 (1993), 449-489.

[10] V. Manuilov, K. Thomsen, Asymptotically split extensions and E-theory, Algebra i Analiz 12 (2000) No. 5, 142-157 (in Russian); English translation: St. Petersburg Math. J. 12 (2001), 819-830.

[11] V. M. Manuilov, K. Thomsen, The Connes-Higson construction is an isomorphism, J. Funct. Anal. 213 (2004), 154-175.

[12] V. Manuilov, K. Thomsen, On the asymptotic tensor $C^{*}$-norm, Preprint.

$[13]$ N. Ozawa, An application of expanders to $\mathbb{B}\left(l_{2}\right) \otimes \mathbb{B}\left(l_{2}\right)$, J. Funct. Anal. 198 (2003), 499-510.

[14] A. Valette, Minimal projections, integrable representations and property T, Arch. Math. 43 (1984), 397-406.

[15] D. Voiculescu, On the non-commutative Weyl-von Neumann theorem, Rev. Roumaine Math. Pures. Appl. 21 (1976), 97-113.

[16] P.S. Wang, On isolated points in the dual space of locally compact groups, Math. Ann. 218 (1975), $19-34$.

[17] S. Wassermann, Liftings in $C^{*}$-algebras: a counterexample, Bull. London Math. Soc. 9 (1976), 201202.

[18] S. Wassermann, Tensor products of free group $C^{*}$-algebras, Bull. London Math. Soc. 22 (1990), $375-380$.

[19] S. Wassermann, $C^{*}$-algebras associated with groups with Kazhdan's property T, Ann. Math. 134 (1991), 423-431.

[20] S. Wassermann, Exact $C^{*}$-algebras and related topics, Lecture Notes Series 19, Seoul National Univ., 1994.

Department of Mechanics and Mathematics, Moscow State University, Leninskie Gory, Moscow, 119992, Russia

E-mail address: manuilov@mech.math.msu.su

imf, Department of Mathematics, Ny Munkegade, 8000 Aarhus C, Denmark

E-mail address: matkt@imf.au.dk 